EPJ Web of Conferences 60, 13011 (2013)

DOI: $10.1051 /$ epjconf/20136013011

(C) Owned by the authors, published by EDP Sciences, 2013

\title{
Recent photon physics results from the ALICE experiment at the LHC
}

\author{
Nicolas Arbor $^{1, a}$ for the ALICE Collaboration \\ ${ }^{1}$ LPSC, Université Joseph Fourier Grenoble1, CNRS/IN2P3, Institut Polytechnique de Grenoble, 53 rue des Martyrs, Grenoble, \\ France
}

\begin{abstract}
We present an overview of the photon analysis in $\mathrm{pp}$ and $\mathrm{Pb}-\mathrm{Pb}$ collisions with data taken by the ALICE experiment at the LHC. The ALICE detectors reconstruct photons by using the two electromagnetic calorimeters (photon spectrometer, sampling calorimeter) and central tracking systems for photon converted $\mathrm{e}^{+} \mathrm{e}^{-}$pairs in the material of the inner ALICE layers. In $\mathrm{Pb}-\mathrm{Pb}$ collisions the direct photon calculations underpredict the data below $4 \mathrm{GeV} / \mathrm{c}$ where it is expected to have a contribution from thermal radiations. The direct photon measurement also shows evidence for a non-zero elliptic flow for $1<p_{\mathrm{T}}<3 \mathrm{GeV} / \mathrm{c}$. The nuclear modification factor of the $\pi^{0}$ production at different collision centralities shows a clear pattern of strong suppression in a hot QCD medium with respect to pp collisions. Finally, parton fragmentation following hard collisions is investigated by correlating high momentum direct photons and charged hadrons with the goal of revealing new insights into medium effects in the QGP.
\end{abstract}

\section{Introduction}

Depending on their energy and their production mechanism, photons probe different properties of the strongly interacting matter produced in heavy ions collisions. Neutral meson spectra, reconstructed via their two-photon decays, are considered to be sensitive to the medium transport properties. Hard partons, produced in the initial stages of heavy-ions collisions, lose energy via collisional and radiative (gluon emissions) mechanisms when traversing the dense and strongly interacting medium. One of the consequences of the parton energy loss is the suppression of high energy hadrons when comparing hadron production yields between heavy-ion and pp collisions. To quantify medium effects, the nuclear modification factor $R_{\mathrm{AA}}$ is calculated, defined as the ratio of hadron transverse momentum spectra, in our case $\pi^{0}$, between $\mathrm{Pb}-\mathrm{Pb}$ collisions scaled by the number of binary nucleon-nucleon collisions $\left\langle N_{\text {coll }}\right\rangle$ and pp collisions,

$$
R_{\mathrm{AA}}=\frac{1}{\left\langle N_{\mathrm{coll}}\right\rangle} \frac{d^{2} N_{\pi^{0}}^{\mathrm{AA}} d y d p_{\mathrm{T}}}{d^{2} N_{\pi^{0}}^{\mathrm{pp}} d y d p_{\mathrm{T}}}
$$

where the number of binary collisions is obtained from the Glauber model, as the product of the nuclear overlap function in the inelastic nucleon-nucleon cross section. If no nuclear modification is present, $R_{\mathrm{AA}}$ is unity.

Decay photons from neutral mesons are also basic ingredients in the extraction of direct photons, as they are dominant sources of the photons from hadron decays. In pp collisions direct photons spectra can be used to constrain the gluon parton distribution function (PDF) which is involved at the leading order in one of the direct photons production mechanism (Compton diffusion) while DrellYann process and deep inelastic scattering only involve gluon PDF at the next-to-leading order. In heavy-ion collisions, direct photons are one of the most promising probes to study the thermal behaviour of the plasma. During the QGP evolution, photons should be produced by the scattering of hard partons traversing the medium, as well as by the scattering of thermalized partons. Thermal radiation of the medium should lead to an excess of direct photon production in a $p_{\mathrm{T}}$ range $1-10 \mathrm{GeV} / \mathrm{c}$. Additional constraints on their production time can be access measuring the direct photon flow $v_{2}$.

In addition, direct photons can be used to perform a tomography of the initial state of the collision. At high $p_{\mathrm{T}}$ direct photons provide invaluable information on the medium interaction with quarks through gamma-hadron (jet) correlations. Direct photons, produced in Compton and annihilation QCD leading order processes, are associated to a jet in opposite azimuthal direction. By correlating the direct photon with hadrons in the opposite direction, one can study jet properties in $\mathrm{pp}$ and $\mathrm{Pb}-\mathrm{Pb}$ collisions in a transverse momentum range below $50 \mathrm{GeV} / \mathrm{c}$ where the full jet reconstruction is challenging because of the relatively large contribution from the underlying event. Such processes are tagged experimentally identifying leading isolated photons and their correlated associated hadrons. The jet fragmentation is estimated from the hadron and the photon $p_{\mathrm{T}}$ and azimuthal angle $\Delta \Phi$ via the imbalance parameter $x_{\mathrm{E}}$ :

$$
x_{\mathrm{E}}=\frac{-p_{\mathrm{T}}^{h} \cdot p_{\mathrm{T}}^{\gamma}}{\left|p_{\mathrm{T}}^{\gamma}\right|^{2}}=-\frac{p_{\mathrm{T}}^{h}}{p_{\mathrm{T}}^{\gamma}} \cos (\Delta \Phi)
$$

\footnotetext{
ae-mail: arbor@lpsc.in2p3.fr
} 


\section{Photon Reconstruction}

The ALICE experiment has the unique capability to measure photons and neutral mesons in three different subsystems : via electromagnetic shower characteristics with PHOS and EMCAL calorimeters, and via photon conversion in the inner material reconstructing $\mathrm{e}^{+} \mathrm{e}^{-}$pairs with the central tracking system. The three different methods have different systematics and therefore provide valuable cross checks with useful $p_{\mathrm{T}}$ overlap over $1-50 \mathrm{GeV} / \mathrm{c}$.

The PHOS detector is a lead tungstate $\left(\mathrm{PbWO}_{4}\right)$ crystals electromagnetic calorimeter. It subtends a pseudorapidity range $|\eta|<0.13$ and an azimuthal range $\Delta \phi=$ $60^{\circ}$. The EMCAL detector is a $\mathrm{Pb}$-scintillator sampling electromagnetic calorimeter that covers $\Delta \phi=100^{\circ}$ in the azimuthal angle and $|\eta| \leq 0.7$ in pseudo-rapidity for the runs taken in 2011, and increased to $\Delta \phi=107^{\circ}$ for 2012 runs. The inner tracking system (ITS) and the time projection chamber (TPC) are the main tracking devices of the ALICE experiment. They are located in the central barrel. The ITS consists of six layers equipped with silicon pixel detectors (SPD), silicon strip detectors (SSD) and silicon drift detectors (SDD). The two innermost cover a pseudorapidity range of $|\eta|<2$ and $|\eta|<1.4$ respectively. The TPC is a large $\left(85 \mathrm{~m}^{3}\right)$ cylindrical drift detector filled with $\mathrm{Ne} / \mathrm{CO}_{2} / \mathrm{N}_{2}(85.7 / 9.5 / 4.8)$ gas mixture. Its acceptance is $|\eta|<0.9$ over the full azimuthal angle. Electron-positron pairs from photon conversions between the beam pipe and the middle of the time projection chamber can be detected with the TPC. The detector material in this range $(11.4 \%$ of a radiation lenght $\mathrm{X}_{0}$ ) corresponds to a photon conversion probability of $\mathrm{p}^{\text {conv }} \approx 8.5 \%$. The centrality of heavyion collision is determined based on the sum of amplitudes measured by the two forward scintillator hodoscopes VZERO that cover $2.8<\eta<5.1$ and $-3.7<\eta<-1.7$.

\section{Analysis Results}

The Figure 1 shows the combined invariant $\pi^{0}$ cross section measured in pp collisions from PHOS and the conversion methods at $\sqrt{s}=0.9,2.76$ and $7 \mathrm{TeV}$ [1]. The cross section is compared to NLO pQCD calculations, which use the CTEQ6M5 parton disctribution functions and the DSS [3] and BKK [4] fragmentation functions. The theoretical uncertainty of the calculation was estimated by varying the unphysical renormalization $\left(\mu_{\mathrm{R}}\right)$, factorization $\left(\mu_{\mathrm{F}}\right)$ and fragmentation $\left(\mu_{\mathrm{F}}\right)$ scales. The NLO pQCD calculation with the DSS and BKK fragmentation function describes the $\pi^{0}$ spectrum at $\sqrt{s}=0.9 \mathrm{TeV}$, but overestimates the data at $\sqrt{s}=2.76$ and $7 \mathrm{TeV}$ for all choices of the scales. This comparison can help to tune the parton distribution function and the fragmentation function involved in the NLO calculations.

The direct photon analyses are based on about $2 \times$ $10^{7}$ minimum bias $\mathrm{Pb}-\mathrm{Pb}$ collisions at $\sqrt{s_{N N}}=2.76 \mathrm{TeV}$ recorded in 2010 with the ALICE experiment at LHC. The inclusive photon yield $\left(\gamma_{\text {inc }}\right)$ is extracted via the reconstruction of their conversion products. The direct photon

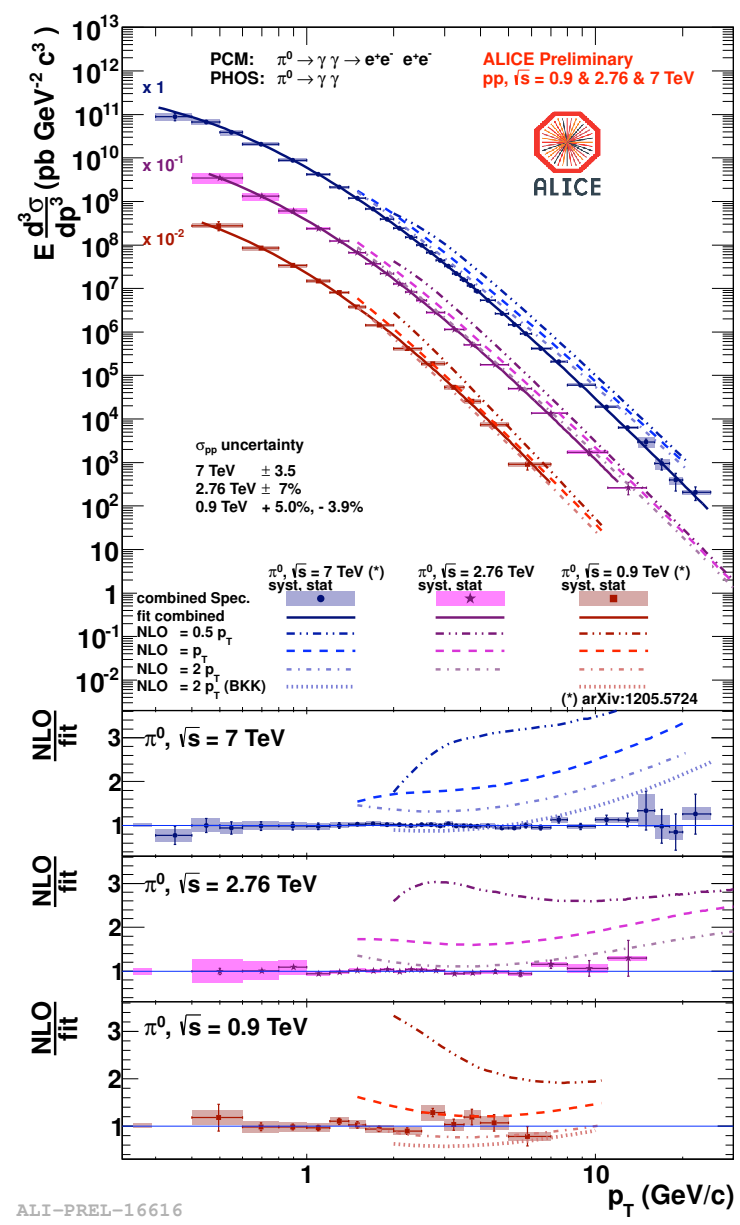

Figure 1. Spectra of $\pi^{0}$ measured in pp collisions at collision energies $\sqrt{s}=0.9,7 \mathrm{TeV}$ [1] and $\sqrt{s}=2.76 \mathrm{TeV}$ [2] compared to NLO pQCD predictions.

spectrum $\left(\gamma_{\text {direct }}\right)$ is then calculated by subtracting all contributions from hadron decays $\left(\gamma_{\text {decay }}\right)$ obtained by a cocktail simulation :

$$
\gamma_{\text {direct }}=\gamma_{\text {inc }}-\gamma_{\text {decay }}=\left(1-\frac{\gamma_{\text {decay }}}{\gamma_{\text {inc }}}\right) \cdot \gamma_{\text {inc }}
$$

Figure 2 shows the direct photon spectrum measured in $\mathrm{Pb}-\mathrm{Pb}$ collisions in the centrality $0-40 \%$ [5]. The $p_{\mathrm{T}}$ spectrum, well described by NLO pQCD predictions above $4 \mathrm{GeV} / c$, shows a significant excess at lower $p_{\mathrm{T}}$ which can be adjusted by an exponential function. The inverse slope parameter of the exponential, $T=\left(304 \pm 51^{\text {stat+sys }}\right) \mathrm{MeV}$, can be interpreted as an effective temperature of the direct photon source integrated over the whole system evolution. The PHENIX collaboration measures an inverse slope parameter of $T=\left(221 \pm 19^{\text {stat }} \pm 19^{\text {syst }}\right) \mathrm{MeV}$ for 0 $20 \% \mathrm{Au}-\mathrm{Au}$ collisions at $\sqrt{s_{N N}}=200 \mathrm{GeV}$ [6][7]. These high effective temperature measurements (compare to the critical temperature $T_{\mathrm{c}}$ ) imply in hydrodynamic models an early production time of direct photons during the system evolution.

Additional constraints on the direct photon production are given by the azimuthal anisotropies of particle emissions studied via a Fourier decomposition with harmonic 


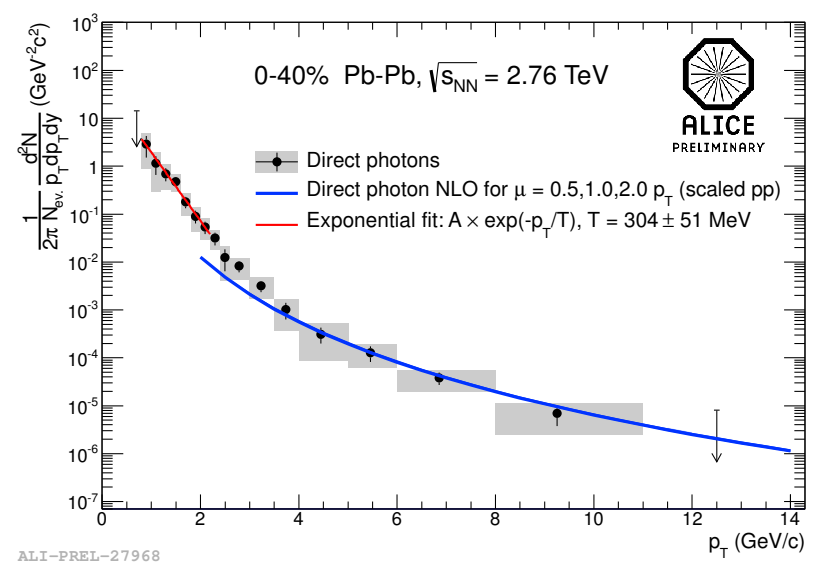

Figure 2. Direct-photon invariant yield in $\mathrm{Pb}-\mathrm{Pb}$ collisions at $\sqrt{s_{N N}}=2.76 \mathrm{TeV}$ for $0-40 \%$ centrality with NLO pQCD predictions and an exponential fit [5].

coefficients $v_{n}$. The direct photon $v_{2}^{\gamma, \text { dir }}$ is calculated from the inclusive photon $v_{2}^{\gamma, \text { inc }}$ by a subtraction of decay photon $v_{2}^{\gamma, \text { decay }}$ obtained by a cocktail simulation parametrized with the measured charged pion $v_{2}^{\pi^{ \pm}}$[8]. Figure 3 shows the measurement of $v_{2}^{\gamma, \text { dir }}$ in $0-40 \% \mathrm{~Pb}-\mathrm{Pb}$ collisions at $\sqrt{s_{N N}}=$ $2.76 \mathrm{TeV}$. A clear evidence for a non-zero $v_{2}^{\gamma, \text { dir }}$ is observed for $1<p_{\mathrm{T}}<3 \mathrm{GeV} / \mathrm{c}$, with a magnitude comparable to that of charged pion $v_{2}^{\pi^{ \pm}}$[9]. The PHENIX collaboration reports similar results [10], which are not described by hydrodynamical calculations including a substantial portion of photons emitted in the plasma phase where flow has not been yet developped [11].

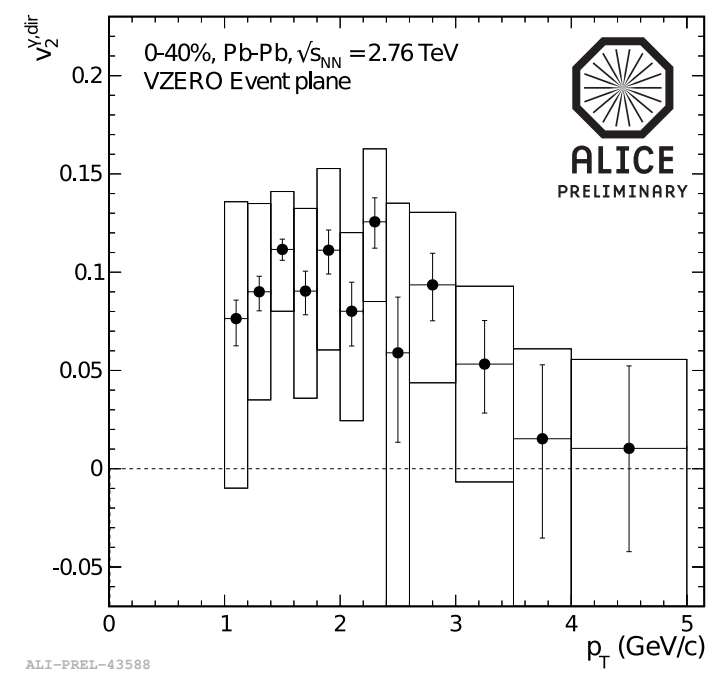

Figure 3. Direct-photon $v_{2}^{\gamma, \text { dir }}$ in $0-40 \% \mathrm{~Pb}-\mathrm{Pb}$ collisions at $\sqrt{s_{N N}}$ $=2.76 \mathrm{TeV}[8]$.

Besides the global properties of the quark-gluon plasma, photons can also be used to probe the hard parton energy loss in the medium. ALICE measured $\pi^{0}$ via the invariant mass of conversion photons in the momentum range $0.5<p_{\mathrm{T}}<10 \mathrm{GeV} / \mathrm{c}$ in the $\sqrt{s_{N N}}=2.76 \mathrm{~Pb}-\mathrm{Pb}$ data collected in 2010 (about $1.3 \times 10^{7}$ events with a centrality between 0 and 80\%) [2]. The derived $R_{A A}$ (Figure 4) shows a suppression of the $\pi^{0}$ production of about $90 \%$ for $p_{\mathrm{T}} \approx 6 \mathrm{GeV} / \mathrm{c}$ in the $20 \%$ of the most central collisions. The nuclear modification factor is compared to several theoretical predictions including WHDG model [12] that takes into account collisional and radiative parton energy loss, Higher-Twist (HT) calculations with $3+1$ dimensional hydrodynamics medium evolution [13] and two calculations (Vitev E-loss) with initial/final state parton energy loss [14]. Depending on the energy loss model, calculations reproduce the strenght of the measured $\pi^{0}$ suppression (WHDG, Higher-Twist, Vitev E-loss), and the centrality dependence (WHDG, Vitev E-loss).

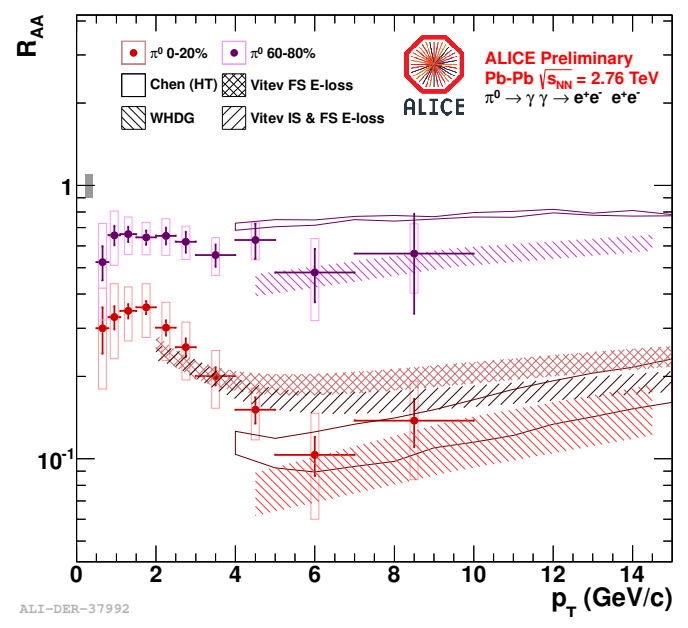

Figure 4. $\pi^{0}$ nuclear modification factor $\left(R_{\mathrm{AA}}\right)$ measured in the $20 \%$ most central and $60-80 \%$ peripheral $\mathrm{Pb}-\mathrm{Pb}$ collisions compared to several theoretical predictions [2].

In contrast to partons, direct photons are not colored objects and, hence, escape almost unmodified from the medium. Photons emitted from such processes may be used to estimate the initial transverse momentum of the recoil parton, and to approximate the parton fragmentation function by measuring photon-hadron correlations (equation 2). These tomographic studies of the medium using parton energy loss in heavy-ion collisions requires first detailed measurement of direct photon-hadron correlations in pp collisions. Direct photon identification is based both on the shape of the electromagnetic shower produced in calorimeters and on an isolation criteria [15]. Figure 5 shows the $x_{E}$ distribution of isolated photon-hadron correlations extracted for a $p_{\mathrm{T}}$ range from 8 to $25 \mathrm{GeV} / \mathrm{c}$ from $2011 \sqrt{s}=7 \mathrm{TeV}$ pp collisions [16].

\section{Summary and Outlook}

The ALICE experiment uses three different techniques for photon reconstruction : two electromagnetic calorimeters (photon spectrometer, sampling calorimeter) and the central tracking systems for photon converted $\mathrm{e}^{+} \mathrm{e}^{-}$pairs. The measured spectrum of direct photons shows an enhancement for the $0-40 \%$ most central collisions, which can not 


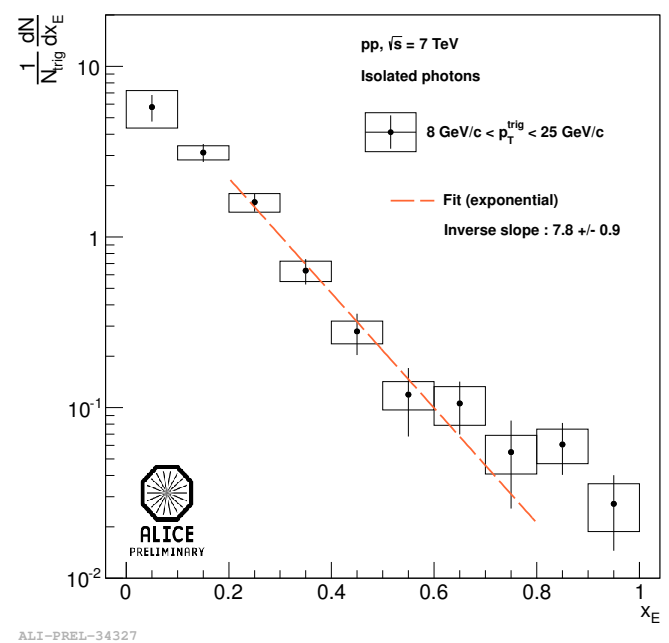

Figure 5. $x_{E}$ distribution of isolated $\gamma$-hadron correlations in $\sqrt{s}$ $=7 \mathrm{TeV}$ pp collisions fitted by an exponential in the $x_{\mathrm{E}}$ range [0.2-0.8] [16].

be described by next-to-leading-order perturbative QCD below $4 \mathrm{GeV} / \mathrm{c}$. The inverse slope parameter of an exponential fit is extracted as $T=\left(304 \pm 51^{\text {stat+sys }}\right) \mathrm{MeV}$. The elliptic flow of direct photons provides evidence for a non-zero $v_{2}^{\gamma, \text { dir }}$ for $1<p_{\mathrm{T}}<3 \mathrm{GeV} / \mathrm{c}$. The observed magnitude, similar to the observed charged pion $v_{2}^{\pi^{ \pm}}$, might lend support for a significant emission from late stages of the medium evolution. The $\pi^{0} R_{\mathrm{AA}}$, measured in the range $0.5<p_{\mathrm{T}}<10 \mathrm{GeV} / \mathrm{c}$, shows a strong suppression that can be used to tune parton energy loss models. On-going analyzes using ALICE calorimeters should help to enlarge the actual $p_{\mathrm{T}}$ range. Finally, photon-hadron correlations can help to understand the parton energy loss mechanism by reconstructing the parton fragmentation function. The $x_{\mathrm{E}}$ distribution measured in pp collisions at $7 \mathrm{TeV}$ provides an essential baseline for the on-going $\mathrm{Pb}-\mathrm{Pb}$ analysis.

\section{References}

[1] B. Abelev et al. [ALICE Collaboration], Phys. Lett. B 717, 162-172 (2012)

[2] D. Peresunko (for the ALICE Collaboration), Nuclear Physics A 904-905, 755c-758c (2013)

[3] D. de Florian, R. Sassot, M. Stratmann, Phys. Rev. D 75 (2007)

[4] J. Binnewies, B.A. Kniehl, G. Kramer, Z. Phys. C 65 (1995)

[5] M. Wilde (for the ALICE Collaboration), Nuclear Physics A 904-905, 573c-576c (2013)

[6] PHENIX Collaboration, Phys.Rev.Lett.94:232301 (2005)

[7] PHENIX Collaboration, Phys.Rev.Lett.104:132301 (2010)

[8] D. Lohner (for the ALICE Collaboration), arXiv : 1212.3995 (2012)

[9] K. Aamodt et al. (ALICE Collaboration), Phys. Rev. Lett. 105252302 (2010)

[10] A. Adare et al. (PHENIX Collaboration), Phys.Rev.Lett. 109122302 (2012)

[11] H. Holopainen, S. Rasanen and K. Eskola, Phys.Rev. C84 064903 (2011)

[12] W.A. Horowitz, Int.J.Mod.Phys.E16, 2193 (2007)

[13] X.-F. Chen et al.,Phys.Rev.C84, 034902 (2011)

[14] R. Sharma et al., Phys.Rev. C80, 054902 (2009). R.B. Neufeld et al., Phys.Lett. B704, 590 (2011)

[15] A. Casanova and G. Conesa, ALICE-INT-2009-002 (2009)

[16] N. Arbor (for the ALICE Collaboration), Nuclear Physics A 904-905, 697c-700c (2013) 